\title{
Minerva: using a software program to improve resident performance during independent call (Withdrawal Notice)
}

Jason Itri, Regina Redfern, Tessa Cook, Mary Scanlon

Jason N. Itri, Regina O. Redfern, Tessa Cook, Mary H. Scanlon, "Minerva: using a software program to improve resident performance during independent call (Withdrawal Notice)," Proc. SPIE 7628, Medical Imaging 2010: Advanced PACS-based Imaging Informatics and Therapeutic Applications, 76280T (11 March 2010); doi: 10.1117/12.845430 


\section{Minerva: using a software program to improve resident performance during independent call (Withdrawal Notice)}

Proc. SPIE 7628, 76280T (2010); http://dx.doi.org/10.1117/12.845430

Online Publication Date: 11 March 2010

Retracted from Publication: 5 May 2010

Conference Date: Wednesday 17 February 2010

Conference Location: San Diego, California, USA

Conference Title: Medical I maging 2010: Advanced PACS-based Imaging Informatics and Therapeutic Applications

Conference Chairs: Brent J. Liu, William W. Boonn

Jason N. Itri, Regina O. Redfern, Tessa Cook, and Mary H. Scanlon

Univ. of Pennsylvania Hospital (USA)

This paper was presented at the SPIE conference indicated above and has been withdrawn from publication at the request of the authors. 